Special issue of the International Conference on Computational and Experimental Science and Engineering (ICCESEN 2014)

\title{
Prediction Methodology of Electromagnetic Emission for Integrated Circuits
}

\author{
M.E. BAŞAK ${ }^{a, *}$ AND A. KUNTMAN ${ }^{b}$ \\ ${ }^{a}$ Yildiz Technical University, Faculty of Naval Architecture and Maritime, Istanbul 34349, Turkey \\ ${ }^{b}$ Istanbul University, Faculty of Engineering, Department of Electrical \& Electronics Engineering, \\ 34850, Avc1lar, Istanbul, Turkey
}

\begin{abstract}
The main objective of this paper is to define a new method which is able to predict the electromagnetic emission of integrated circuits. In this study, a test circuit including the MC9S12XMAG-family microcontroller was designed and measurements were made to confirm the applied measurement techniques. Moreover, internal current was obtained from the measured external current which is described as a dynamic current activity on the die. The input impedance measurements were performed in the frequency range from $1 \mathrm{MHz}$ to $2 \mathrm{GHz}$. The external current was measured by the spectrum analyzer has been used to obtain the internal current of the die. Extracted passive distribution network and internal activity component values have been given to obtain the integrated circuit emission model. The magnetic field is simulated at different frequencies and heights over the circuit under test. The model allows simulating the same magnetic field radiated by the component under test with the suficient accuracy for the application at any distance from the device under test. The model considers the component like a "black box", so that it can be applied to any passive or active component.
\end{abstract}

DOI: 10.12693/APhysPolA.128.B-248

PACS: 52.25 .Os

\section{Introduction}

Electromagnetic compatibility (EMC) is the ability of an electrical or electronic product or system can operate accurately in the intended environment without degradation of performance due to unintentional electromagnetic interactions. Daily a new electronic product is entering into our lives. Increasing integration of electronic products and/or systems are becoming more complex nowadays. Increasing the power and frequency range of electronic devices are creating a more severe and uncontrollable electromagnetic environment. It is difficult to operate in the intended environment without causing performance degradation due to unintentional electromagnetic effects.

The lately developed integrated circuits (ICs) generated the amount of parasitic emissions due to advances in digital and semiconductor technology with higher clock speeds, more complex circuits, and upper dynamic current consumptions [1].

Highest in a number of the EMC related problem concentrated on the external part of the IC, despite the fact that, ICs have important functions in many cases of the electromagnetic compatibility of an electronic system. In this study, internal part of the IC core has been focused on. EMC is generally checked at the end of the design cycle, before the IC is placed on the printed circuit board. If the emission level is higher than the expected, the design will be done again and this will be

*corresponding author; e-mail: mebasak@yildiz.edu.tr caused by loss of time and money. For that reason, a model is needed to predict the EM compliance precisely and swiftly. Various electromagnetic models have been generated to predict the behavior of the ICs in an electromagnetic environment [1].

\section{Measurement and modelling}

In this study MC9S12XMAG microcontroller, produced by Freescale semiconductor, was used. This 16-bit microcontroller has been widely used in recent automotive electronic systems. Designed and measured MC9S12XMAG microcontroller have been shown in Fig. 1. This microcontroller fabricated in $0.25 \mu \mathrm{m}$ CMOS technology. It has nine pairs of power and ground pins to supply the I/O ports, the A/D converter, the oscillator, the phase locked loop (PLL), and the digital core. The pinout of the microcontroller mounted in a thin quad flat patch (TQFP) package with 144 pins.

Because of practical reasons, we measured $S$ parameters; eventually we converted the results to $Z$ parameters. Once the measurements had been done, the impedance model was extracted. It represents a first order resonance, obtaining the global resistance value $R$ at the frequency resonance. But before setting up the impedance model, we should remove the effects of the PCB board, conductive path between the power supply pin and SMA connector, and SMA connector. De-embedding is a mathematical method that removes the undesirable effects of the structure that are embedded in the measured data by deducting their contribution. Traces on the board can cause a larger measured impact than the device under test itself [2]. 


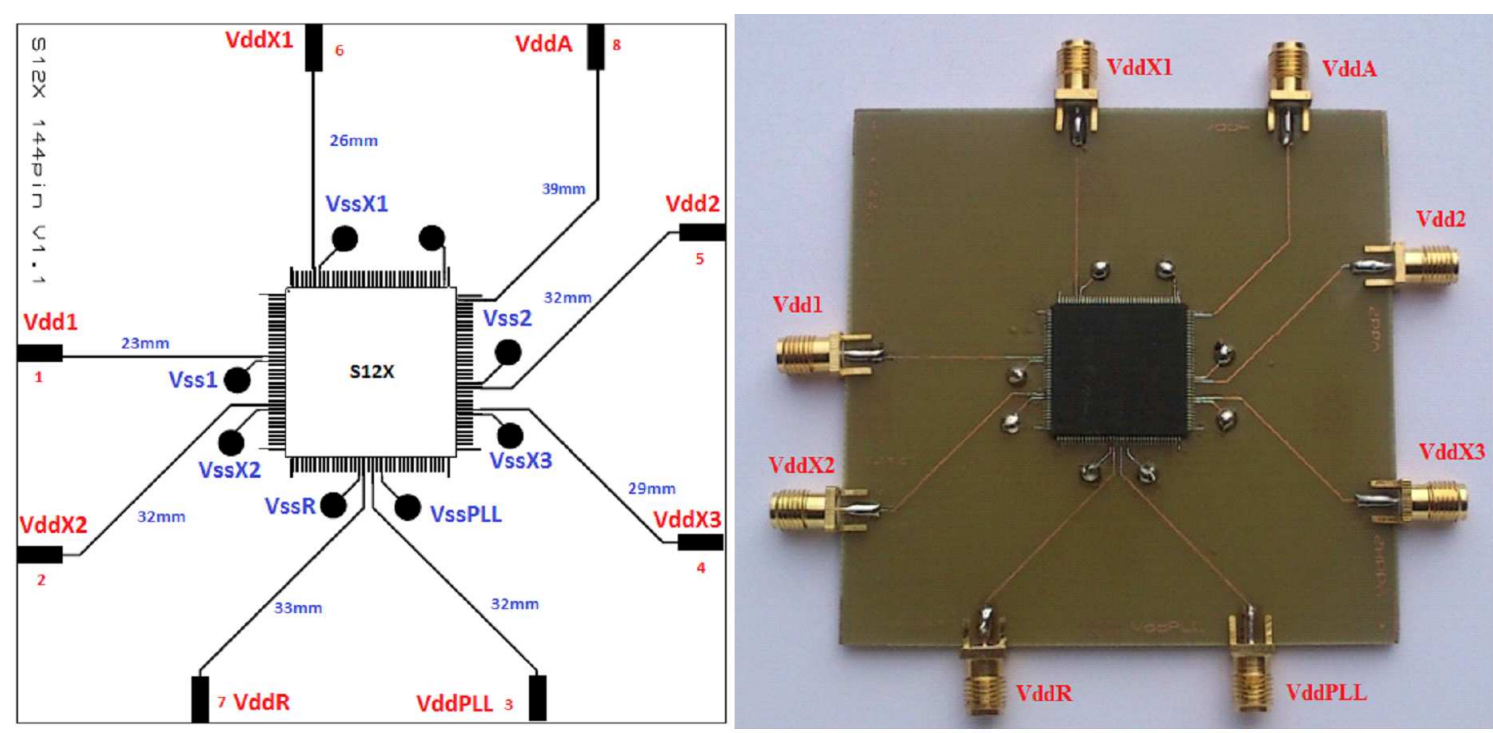

Fig. 1. Designed and measured MC9S12XMAG microcontroller.

$S$-parameters were measured by network analyzer and then the results translated into impedance $Z$ from which $R, L$, and $C$ elements were tuned manually. Then, the impedance of the SMA connector, path and board between the power supply pin and SMA connector was deleted by the de-embedding technique. After measurements of $S$ parameters of the circuits in the specified frequency range were done from $1 \mathrm{MHz}$ to $2 \mathrm{GHz} ; Z$ parameters were obtained from $S$ parameters.

Most of the time, internal activity (IA) is not accessible directly. The transfer function of PDN has been already extracted and the current flowing externally, $I_{\text {ext }}$ has been measured. Depending on which analysis is needed, IA can be described either in the time or in the frequency domain. The current was measured by Spectrum Analyser has been used to obtain the external current. After the measurement, the results were calibrated by subtracting the effect of the amplifier. The effect of the amplifier has been found by Vector Network Analyser.

From the set of equations, it is possible to obtain the radiation at a certain point of space $(M)$, by summing the emissions of all individual dipoles as shown in Fig. 2.

$$
\begin{aligned}
H_{x} & =\frac{I_{0}}{2 \pi R^{2}} \Delta \ell\left(\frac{z-z_{0}}{R}+\frac{\mathrm{j} \omega\left(z-z_{0}\right)}{c}\right) \sin \theta, \\
H_{y} & =\frac{-I_{0}}{2 \pi R^{2}} \Delta \ell\left(\frac{z-z_{0}}{R}+\frac{\mathrm{j} \omega\left(z-z_{0}\right)}{c}\right) \cos \theta, \\
H_{z} & =\frac{I_{0}}{2 \pi R^{2}} \Delta \ell\left[-\left(\frac{x-x_{0}}{R}+\frac{\mathrm{j} \omega\left(x-x_{0}\right)}{c}\right) \sin \theta\right. \\
& \left.+\left(\frac{y-y_{0}}{R}+\frac{\mathrm{j} \omega\left(y-y_{0}\right)}{c}\right) \cos \theta\right], \\
R & =\sqrt{\left(x-x_{0}\right)^{2}+\left(y-y_{0}\right)^{2}+\left(z-z_{0}\right)^{2}} .
\end{aligned}
$$

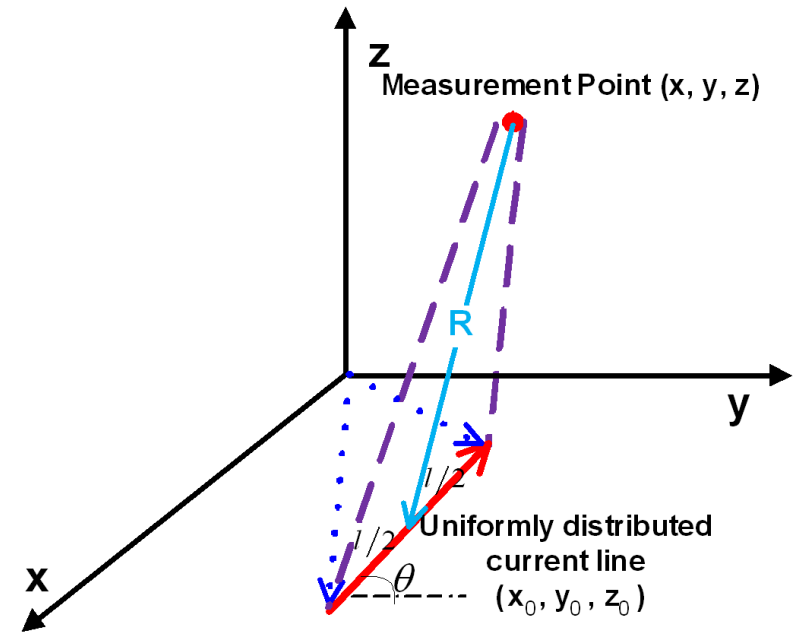

Fig. 2. Uniformly distributed current line.

In this proposed method, a set of uniformly RF distributed current lines, in the $x-y$ plane is used. Some parameters of the model are fixed, namely the number, length and position of the uniformly distributed current lines. Figure 3 below shows the equivalent representation of the DUT in terms of uniformly RF distributed current lines.

The magnetic field plots show good correlation between the fields radiated in all cases and we can conclude that the integrated model is accurate to our design and meets our specifications to a good extent, even though we do not get $100 \%$ accurate results. But the best aspect is that the modelled field correlates with integrated model's field and the integration process is good. We have a few errors that occur between the simulations. 


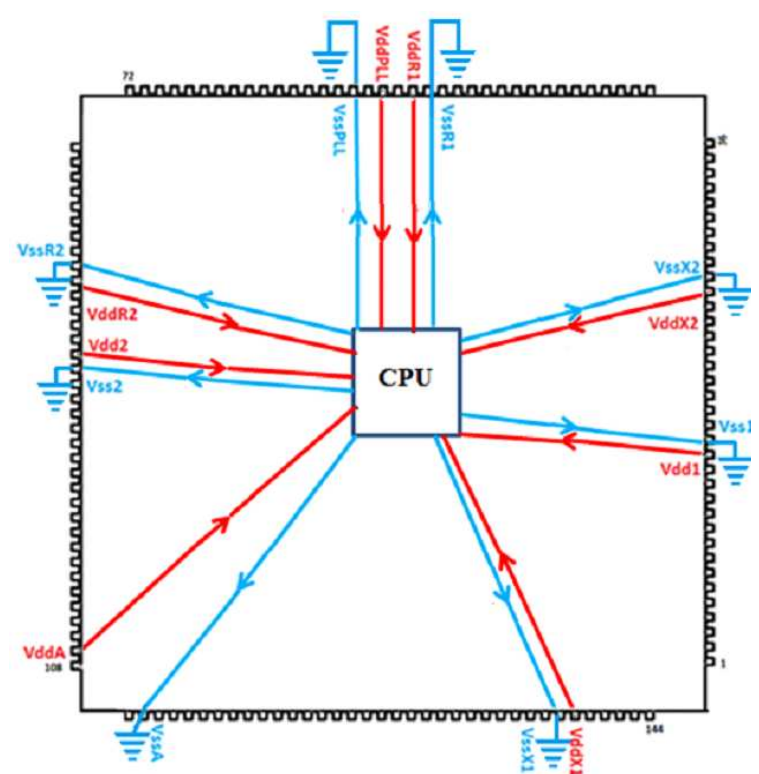

Fig. 3. The proposed model of the IC.

\section{Conclusion}

In this paper, the power supply conducted emissions of an IC have been evaluated. The power distribution network is characterized by self and transfer impedances. Good agreement between measured and simulated impedances was found. Extracted PDN and IA component values have been given to obtain the integrated circuit emission model. This model will be used to evaluate electromagnetic behavior and performance of electronic equipment. In the future, the exact model will be designed and model results will be compared with the near field measurements for specified frequencies.

\section{References}

[1] S.B. Dhia, M. Ramdani, E. Sicard, Electromagnetic compatibility of integrated circuits - techniques for low emission and susceptibility, Springer-Verlag, New York 2006.

[2] M.E. Başak, A. Kuntman, Tümdevrelerin ELECO 2012, Elektrik Elektronik Bilgisayar Mühendisliği Semp., Bursa 2012, p. 319, (in Turkish). 